\title{
Dynamics and instability of false vacuum bubbles
}

\author{
Anthony Aguirre \& Matthew C. Johnson \\ Department of Physics, University of California, Santa Cruz, California 95064, USA \\ aguirre@scipp.ucsc.edu; mjohnson@physics.ucsc.edu
}

(Dated: October 8, 2018)

\begin{abstract}
This paper examines the classical dynamics of false vacuum regions embedded in surrounding regions of true vacuum, in the thin-wall limit. The dynamics of all generally relativistically allowed solutions - most but not all of which have been previously studied - are derived, enumerated, and interpreted. We comment on the relation of these solutions to possible mechanisms whereby inflating regions may be spawned from non-inflating ones. We then calculate the dynamics of first order deviations from spherical symmetry, finding that many solutions are unstable to such aspherical perturbations. The parameter space in which the perturbations on bound solutions inevitably become nonlinear is mapped. This instability has consequences for the Farhi-Guth-Guven mechanism for baby universe production via quantum tunneling.
\end{abstract}

PACS numbers: $98.80 . \mathrm{Hw}, 98.80 . \mathrm{Cq}$

\section{INTRODUCTION}

Nearly two decades ago, a series of papers [1, 2, 3] began to investigate the possibility of creating an inflationary universe "in a laboratory" - that is, inside a surrounding region of much lower vacuum energy. The spacetime of such a "bubble universe" was modeled as a spherically symmetric de Sitter region (the false vacuum) joined to a surrounding Schwarzschild geometry (the true vacuum) by an infinitesimally thin domain wall.

These studies found that the inflating (false vacuum) region could not avoid collapse unless either the null energy condition or cosmic censorship were violated in the full spacetime 1], but that the creation of an enduring inflating region might be possible via quantum tunneling [2, 3]. In this picture, henceforth denoted the Farhi-Guth-Guven (FGG) mechanism, a classically constructable expanding bubble, which would classically re-collapse (both from the inside and outside perspective [4]), instead tunnels to a new solution in which the inflating interior expands forever, while an outside observer sees a black hole [35]. The probability of this occurring can be calculated using the techniques of semiclassical quantum gravity, and is extraordinarily small.

Though nearly-miraculous, this process has garnered new interest recently, primarily because of evidence that our universe may have a fundamental positive cosmological constant. If so, it will asymptotically approach everlasting equilibrium as de Sitter spacetime. Given eternity, even the most unlikely process - such as the creation of inflating bubble universes - will eventually occur. Taking this one step further, our observable universe could in fact be such a bubble universe, which arose from equilibrium de Sitter space and is currently returning to it. This would realize the old idea of Boltzmann that the universe is fundamentally in equilibrium, but that extremely rare downward fluctuations in entropy periodically occur and allow the transitory existence of non-equilibrium regions that see entropy steadily increasing.

The classic problem with this idea was pointed out in its new context by Dyson, Susskind, \& Kleban [5]: if our observable universe resulted from a downward entropy fluctuation that evolved with increasing entropy to the present time, then it is vastly more likely for this to have occurred by a fluctuation to our observable universe ten minutes ago (replete with incoming photons and memories in our brains to convince us that it is older) than by a fluctuation all the way to the much lower entropy corresponding to inflation [36]. Albrecht and Sorbo [6], however, argue that inflation might avoid this problem by turning a tiny region of low entropy density into a very large one. Their calculation shows that the FGG mechanism requires a much smaller entropy fluctuation than directly creating the large post-reheating region that will result from it, thus resolving the paradox.

There may, however, be reasons to doubt that the creation of small regions of false vacuum and subsequent tunneling are plausible events. First, there is no regular instanton describing either the nucleation of small regions of false vacuum or the gravitational tunneling event; there $i s$ such an instanton describing tunneling to falsevacuum, but over a huge region, larger than the truevacuum horizon (see, e.g., [10, 11]). Second, Banks [12] has pointed out that it is hard to understand the tunneling process holographically - it appears that the inflating region inside the bubble has many more states than the black hole it is "contained in" (see also [13]). Third, Dutta \& Vachaspati [14] have recently (and previously in [15]) argued that general causality considerations preclude the formation of a small false-vacuum region inside a large true-vacuum one.

Both in terms of the initial conditions for inflation, and also the more general issue of what processes can lead to transitions between vacuum states (as is important in understanding the string theory "landscape"), it is crucial to understand bubble universes, whether they can form, and with what probabilities. To this question the present paper makes the following contributions: first, we organize and interpret all of the thin-walled, spherically symmetric one bubble spacetimes. We then show 
that expanding false vacuum bubbles are unstable to nonspherical perturbations; if these bubbles start at a sufficiently small initial radius, then they inevitably become nonlinearly aspherical before tunneling might occur in the FGG mechanism. It is unclear in this case whether tunneling to an inflationary universe inside the bubble can occur at all, or with what probability.

The plan of the paper is as follows. In Sec. III the allowed solutions to the junction conditions are enumerated, putting the previous work into context. We provide a concise reference for all of the possible spacetimes with one false-vacuum bubble and arbitrary positive cosmological constant, then discuss the existing, and some new, interpretations of these solutions. In Sec. III) we derive the first order perturbation equations, and demonstrate the existence of an instability in bound solutions. In Sec. IV we integrate the equations to investigate the parameter space for which non-linear perturbations are unavoidable, and we conclude in Sec. $\nabla$

\section{JUNCTION CONDITIONS}

The dynamics of inflating regions has been discussed by a number of authors [4, 16, 17, 18] 37] using the junction condition formalism. These works study a spherically symmetric region of false vacuum (high energy density) in a surrounding region of true vacuum (lower energy density). The wall separating the regions is assumed to be very thin compared to the radius of the region of false vacuum. One can obtain the dynamics of the wall by requiring metric continuity across the wall and then solving Einstein's equations. Under spherical symmetry, the dynamics of the problem then reduce to those of the bubble wall's radius. This radius is a gauge invariant quantity because it simply quantifies the curvature of the bubble wall worldsheet, and any observer can measure it by comparing the normal to the wall at two nearby points.

\section{A. Interior and Exterior Spacetimes}

Let $\Lambda_{+}$be the cosmological constant in the true vacuum region. Then if $\Lambda_{+}>0$, the region is Schwarzschildde Sitter spacetime with metric

$$
\begin{gathered}
d s_{+}^{2}=-a_{\mathrm{sds}} d t^{2}+a_{\mathrm{sds}}^{-1} d r^{2}+r^{2} d \Omega^{2}, \\
a_{\mathrm{sds}}=1-\frac{2 M}{r}-\frac{\Lambda_{+}}{3} r^{2}
\end{gathered}
$$

in the static foliation. Fixing $\Lambda_{+}$, there are then three qualitatively different casual structures characterized by the value of $M$ (see 19]), due to the nature of the three roots of $a_{\mathrm{sds}}(r)$.

For $3 M<\Lambda_{+}^{-1 / 2}$, there are three distinct real roots of

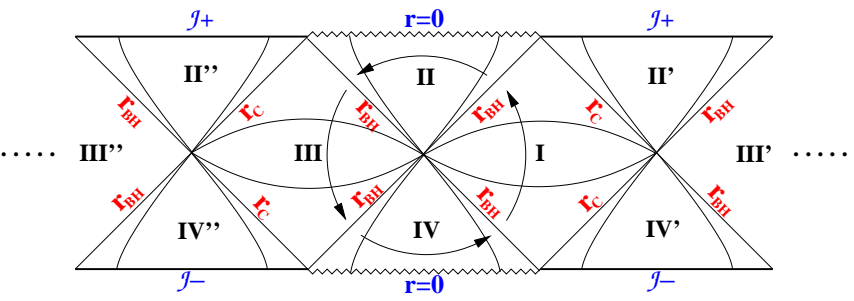

FIG. 1: Conformal diagram of the Schwarzschild de Sitter geometry for $3 M<\Lambda_{+}$.

form:

$$
r_{n}=2\left(\Lambda_{+}\right)^{-1 / 2} \cos \left(\frac{\theta}{3}+\frac{2 \pi n}{3}\right)
$$

where

$$
\cos \theta=-3 M\left(\Lambda_{+}\right)^{1 / 2},
$$

and $\pi<\theta<3 \pi / 2$. We can label them as

$$
r_{B H} \equiv r_{0}, \quad r_{\text {neg }}=r_{1}, \quad r_{c}=r_{2},
$$

And the range of $\theta$ means that they lie in the ranges $r_{\text {neg }}<0<2 M<r_{B H}<3 M<r_{c}$.

The two positive roots correspond to the black hole and cosmological horizons. The conformal diagram for this spacetime is shown in Fig. [1 (See 20] for a demonstration of the explicit form of the metric in global coordinates). Surfaces of constant coordinate time $t$ are drawn, with the circulating arrows denoting the direction of increasing $t$. We will consider region I to be the "causal patch" of a hypothetical observer (i.e., the region lying in both the causal past and causal future of the observer's world line) in what follows.

For $3 M=\Lambda_{+}^{-1 / 2}$, there are also three real roots: a double positive root $r_{h}$ and a negative $r_{\text {neg }}$, given by:

$$
r_{h}=\Lambda_{+}^{-1 / 2}, \quad r_{\text {neg }}=-2 \Lambda_{+}^{-1 / 2} .
$$

This mass is known as the Nariai mass, and in this spacetime there is only one horizon at the positive root. The conformal diagram for this spacetime is shown in Fig. 2 [19]. There is also a time-reverse solution, starting at past null infinity and ending at $r=0$. For $3 M>\Lambda_{+}^{-1 / 2}$, there is one real negative root, and therefore no horizons in the spacetime. The conformal diagram for this case is like figure 2 but with the horizon lines excised.

Inside the false-vacuum region the spacetime is de Sitter space, with metric

$$
\begin{gathered}
d s_{-}^{2}=-a_{\mathrm{ds}} d t^{2}+a_{\mathrm{ds}}^{-1} d r^{2}+r^{2} d \Omega^{2}, \\
a_{\mathrm{ds}}=1-\frac{\Lambda_{-}}{3} r^{2}
\end{gathered}
$$

in the static foliation. Fig. 3 shows the conformal diagram for the de Sitter region. Again, surfaces of constant 


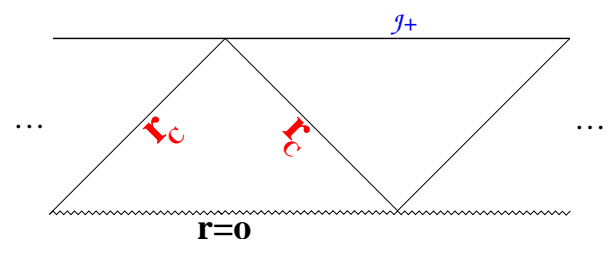

FIG. 2: Conformal diagram for the Schwarzschild de Sitter geometry when $3 M=\Lambda_{+}$.

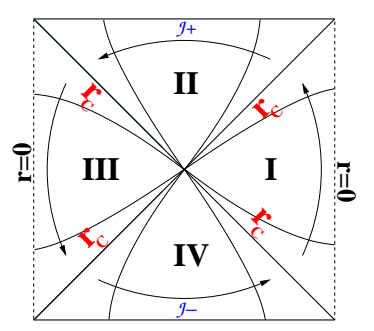

FIG. 3: Conformal diagram for the de Sitter geometry.

coordinate time $t$ are shown, with the arrows denoting the direction of increasing $t$. We consider region III to be the causal patch in which our hypothetical false vacuum observer resides.

\section{B. Equation of Motion}

The bubble wall worldsheet has metric:

$$
d s^{2}=-d \tau^{2}+r(\tau)^{2} d \Omega^{2}
$$

where $\tau$ is the proper time in the frame of the wall, and $(\theta, \phi)$ are the usual angular variables.

The coordinates in the full $4 D$ spacetime are chosen to be Gaussian normal coordinates constructed in the neighborhood of the bubble wall worldsheet. Three of the coordinates are $(\tau, \theta, \phi)$ on the worldsheet, and the fourth, $\eta$, is defined as the proper distance along a geodesic normal to the bubble worldsheet, with $\eta$ increasing in the direction of SdS (true vacuum).

The transformation from the static coordinate systems of $\mathrm{SdS}$ and dS to the Gaussian normal system can be constructed in closed form using the methods of [2], and the full metric takes the form:

$$
d s^{2}=g_{\tau \tau}(\tau, \eta) d \tau^{2}+d \eta^{2}+R(\tau, \eta)^{2} d \Omega^{2},
$$

where $\eta=0$ defines the wall and therefore $g_{\tau \tau}(\tau, 0)=-1$ and $R(\tau, 0)=r(\tau)$.

The energy momentum tensor on the wall is:

$$
T_{\mathrm{wall}}^{\mu \nu}=-\sigma \gamma^{\mu \nu} \delta(\eta)
$$

where $\gamma^{\mu \nu}$ is the metric on the worldsheet of the wall for $\mu=\nu=\tau, \theta, \phi$ and zero otherwise, and $\sigma$ is the energy density of the wall.

Using the metric 8 and the energy-momentum tensor 9 together with the contributions from the dS interior and
SdS exterior in Einstein's equations yields an equation of motion for the bubble wall of [2, 4] :

$$
K_{j}^{i}\left(\eta_{+}\right)-K_{j}^{i}\left(\eta_{-}\right)=-4 \pi \sigma r \delta_{j}^{i},
$$

where $K_{j}^{i}\left(\eta_{ \pm}\right)$is the extrinsic curvature tensor in SdS and dS respectively. In the Gaussian normal coordinates, this takes the form:

$$
K_{i j}=\frac{1}{2} \frac{d}{d \eta} g_{i j}
$$

Evaluating this in metric 8 the $\theta \theta$ and $\phi \phi$ components of Eq. 10] reduce to:

$$
\beta_{\mathrm{ds}}-\beta_{\mathrm{sds}}=4 \pi \sigma r
$$

with the definitions

$$
\beta_{\mathrm{ds}} \equiv-a_{\mathrm{ds}} \frac{d t}{d \tau}, \quad \beta_{\mathrm{sds}} \equiv a_{\mathrm{sds}} \frac{d t}{d \tau} .
$$

Here, $a$ is the metric coefficient in $\mathrm{dS}$ or $\mathrm{SdS}$. The sign of $\beta$ is fixed by the trajectory because $d t / d \tau$ could potentially be positive or negative (motion can be with or against the direction of increasing coordinate time indicated in Fig 1 and 3).

A set of dimensionless coordinates can be defined, in which Eq. 12 can be written as the equation of motion of a particle of unit mass in a one dimensional potential [18]. Let:

$$
z=\left(\frac{L^{2}}{2 M}\right)^{\frac{1}{3}} r, \quad T=\frac{L^{2}}{2 k} \tau,
$$

where $\mathrm{M}$ is the mass appearing in the SdS metric coefficient, and

$$
\begin{gathered}
k=4 \pi \sigma \\
L^{2}=\frac{1}{3}\left[\left|\left(\Lambda_{-}+\Lambda_{+}+3 k^{2}\right)^{2}-4 \Lambda_{+} \Lambda_{-}\right|\right]^{\frac{1}{2}} .
\end{gathered}
$$

With these definitions, Eq. 12 becomes

$$
\left[\frac{d z}{d T}\right]^{2}=Q-V(z),
$$

where the potential $V(z)$ and energy $Q$ are

$$
V(z)=-\left[z^{2}+\frac{2 Y}{z}+\frac{1}{z^{4}}\right],
$$

with

$$
Y=\frac{1}{3} \frac{\Lambda_{+}-\Lambda_{-}+3 k^{2}}{L^{2}}
$$

and

$$
Q=-\frac{4 k^{2}}{(2 M)^{\frac{2}{3}} L^{\frac{8}{3}}} .
$$




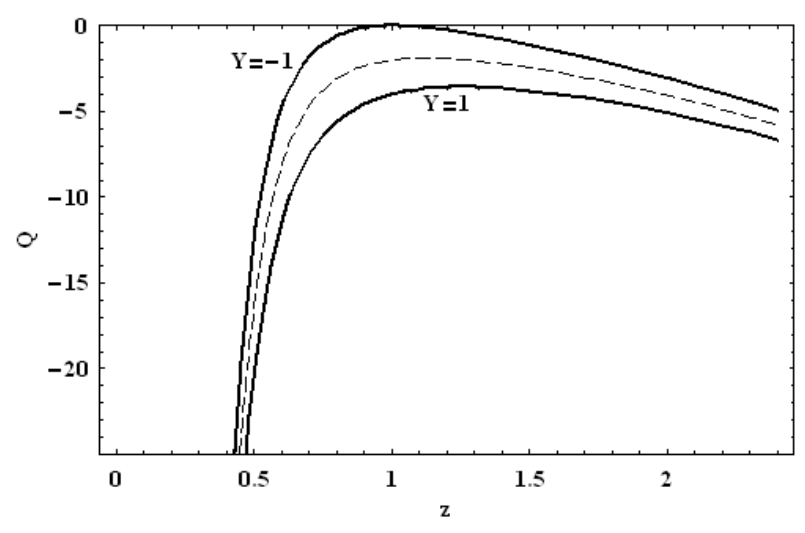

FIG. 4: The potential for various Y.

Note that a small negative $Q$ corresponds to a large mass, so that even between $-1<Q<0$ the mass will vary by many orders of magnitude.

The parameter space allowed by the junction conditions is characterized by the value of the cosmological constant inside and outside the wall. For $0 \leq \Lambda_{+}<\Lambda_{-}$, we have $-1 \leq Y \leq 1$. The maximum $V_{\max }$ of the potential $V(z)$ then satisfies $-2^{5 / 3}-2^{-4 / 3} \leq V_{\max } \leq 0$. The potential curves over the entire range of $Y$ are shown in Fig 4

The interior and exterior cosmological constants can be expressed in terms of $k^{2}$ as $\Lambda_{+}=A k^{2}$ and $\Lambda_{-}=B k^{2}$. With these choices, the dynamics of the bubble wall are entirely determined by $A, B$, and $Q$.

Let us now discuss some realistic values for the parameters in this theory. The interior cosmological constant $\left(\Lambda_{-}=M_{I}^{4} / M_{p l}^{4}\right)$ and the bubble wall surface energy density $\left(k=4 \pi M_{I}^{3} / M_{p l}^{3}\right)$ will be set by the scale of inflation $\left(M_{I}\right)$. The exterior cosmological constant $\left(\Lambda_{+}=M_{\Lambda}^{4} / M_{p l}^{4}\right)$ will be set by a scale $M_{\Lambda}$. These yield

$$
A=\frac{M_{\Lambda}^{4} M_{\mathrm{pl}}^{2}}{(4 \pi)^{2} M_{I}^{6}}, \quad B=\frac{M_{\mathrm{pl}}^{2}}{(4 \pi)^{2} M_{I}^{2}} .
$$

We will consider three representative energy scales, covering the interesting range of energy scales for inflation. For weak scale inflation $(100 \mathrm{GeV}), k \simeq 4 \pi \times 10^{-51}$, $A \simeq 0$, and $B \simeq 10^{32}$. For an inflation scale near the GUT scale $\left(10^{14} \mathrm{GeV} \gg M_{\Lambda}\right)$, we have $k \simeq 4 \pi \times 10^{-15}$, $A \simeq 0$, and $B \simeq 10^{7}$. Near-Planck scale inflation $\left(10^{17}\right.$ $\mathrm{GeV}$ ) yields $k \simeq 4 \pi \times 10^{-6}, A \simeq 0$, and $B \simeq 63$. The most massive bound solution (that which just reaches the top of the potential) is given by converting from $Q$ to $M$ using Eq. 20. This maximal mass is very different in each case, ranging from an ant-mass of $M_{\max } \simeq$ $10^{3} M_{\mathrm{pl}} \simeq 10^{-2}$ grams for $M_{I}=10^{17} \mathrm{GeV}$ to an Earthmass of $M_{\max } \simeq 10^{33} M_{\mathrm{pl}} \simeq 10^{28}$ grams, for $M_{I}=100$ $\mathrm{GeV}$.

\section{Allowed Solutions}

A bubble wall trajectory is characterized by $Q=$ const., and there are three general types:

- Bound solutions with $Q<V_{\max }$. These solutions start at $z=0$, bounce off the potential wall and return to $z=0$.

- Unbound solutions with $Q<V_{\max }$. These solutions start at $z=\infty$, bounce off the potential wall and return to $z=\infty$.

- Monotonic solutions with $Q>V_{\max }$. These solutions start out at $z=0$ and go to $z=\infty$, or execute the time-reversed motion.

The qualitative features of a generic potential can be shown by considering the four illustrative (but unrealistic; see above) cases of $(A=9, B=15)$ shown in Fig. [5 $(A=1, B=6)$ shown in Fig. 6] $(A=1, B=2)$ shown in Fig. 7 and $(A=2.9, B=3)$ shown in Fig. 8 The important features are:

- As one follows a line of constant $Q$ in Fig. [5, 6, 7 and 8 every intersection with the dashed line $Q_{\text {sds }}$ (which is obtained by solving $a_{\mathrm{sds}}=0$ for $Q$ ) represents a horizon crossing in the SdS spacetime (this could either represent the black hole or cosmological horizons).

- Intersections with the dashed line $Q_{\mathrm{ds}}$ (which is obtained by solving $a_{\mathrm{ds}}=0$ for $Q$ ) as one moves along a line of constant $Q$ represent the crossing of the interior dS horizon.

- The vertical line on the right denotes the position at which $\beta_{\mathrm{ds}}$ changes sign. $\beta_{\mathrm{ds}}>0$ if $t_{\mathrm{ds}}$ is decreasing along the bubble wall trajectory and is negative if $t_{\mathrm{ds}}$ is increasing. For there to be a $\beta_{\mathrm{ds}}$ sign change, $Y$ in Eq. 19] must be in the range $-1 \leq Y<0$ [18], which yields the condition that $B>A+3$ for a sign change to occur.

- The vertical dotted line on the left denotes the radius at which $\beta_{\text {sds }}$ changes sign. Recall that $\beta_{\mathrm{sds}}>0$ if $t_{\mathrm{sds}}$ is increasing along the bubble wall trajectory, and $\beta_{\mathrm{sds}}<0$ if it is decreasing. For the parameters in Fig. 5 and 8 or (one can show) whenever $B<3(A-1)$, the sign change occurs to the right of the maximum of $V$. When $B>3(A-1)$ it occurs to the left [38], as shown in Fig. [6 (note that this is the interesting case where $\Lambda_{+} \ll \Lambda_{-}$) and 7

The potential diagram contains all of the information needed to determine the conformal structure of the allowed one-bubble spacetimes. A complete set of the qualitatively different trajectories for arbitrary interior and exterior positive definite cosmological constants (with 


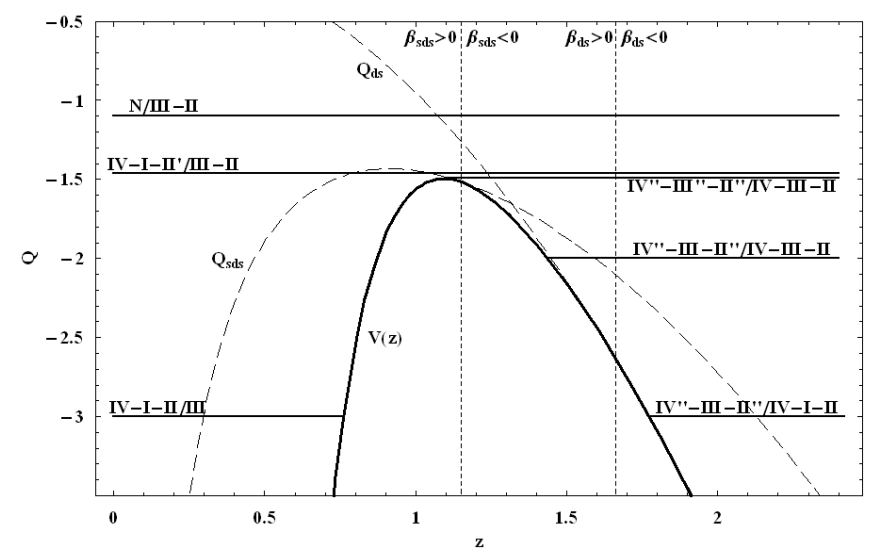

FIG. 5: Potential for $A=9$ and $B=15$. The two dashed lines labeled $Q_{\text {sds }}$ and $Q_{s}$ represent the exterior and interior horizon crossings respectively. The vertical dotted lines denote the regions in which $\beta_{\mathrm{sds}}$ and $\beta_{\mathrm{ds}}$ are positive and negative. Various trajectories are noted.

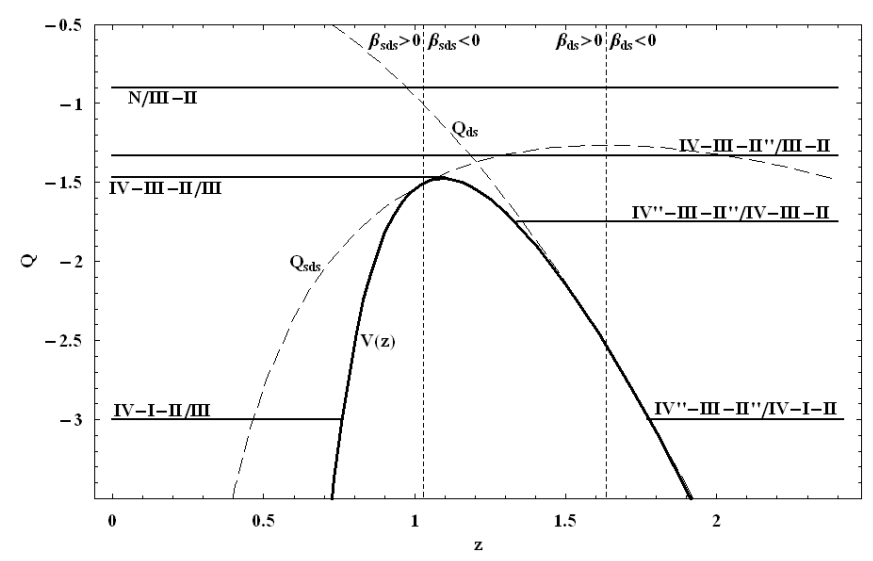

FIG. 6: Potential for $A=1$ and $B=6$. For this choice of parameters, the sign change in $\beta_{\text {sds }}$ occurs to the left of the maximum in the potential. Various trajectories are noted.

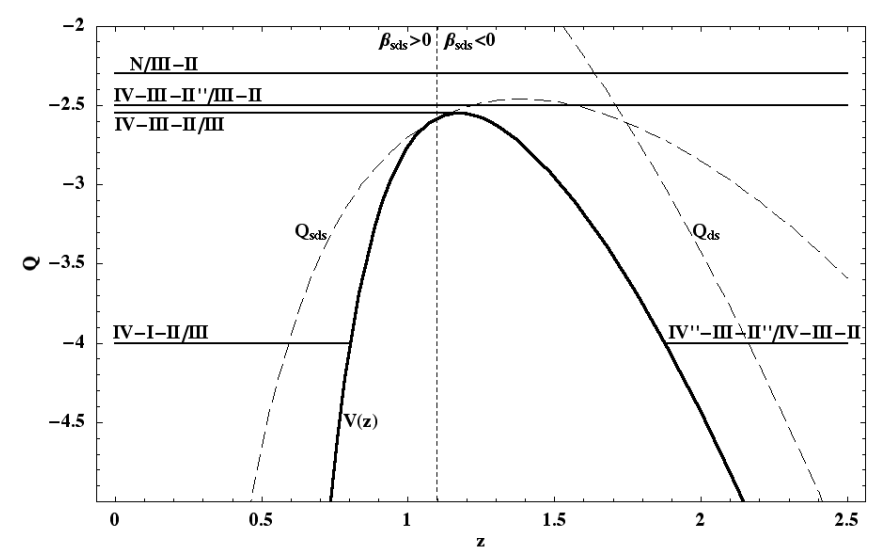

FIG. 7: Potential for $A=1$ and $B=2$. For this choice of parameters, the sign change in $\beta_{\text {sds }}$ occurs to the left of the maximum in the potential and there is no $\beta_{\mathrm{ds}}$ sign change. Various trajectories are noted.

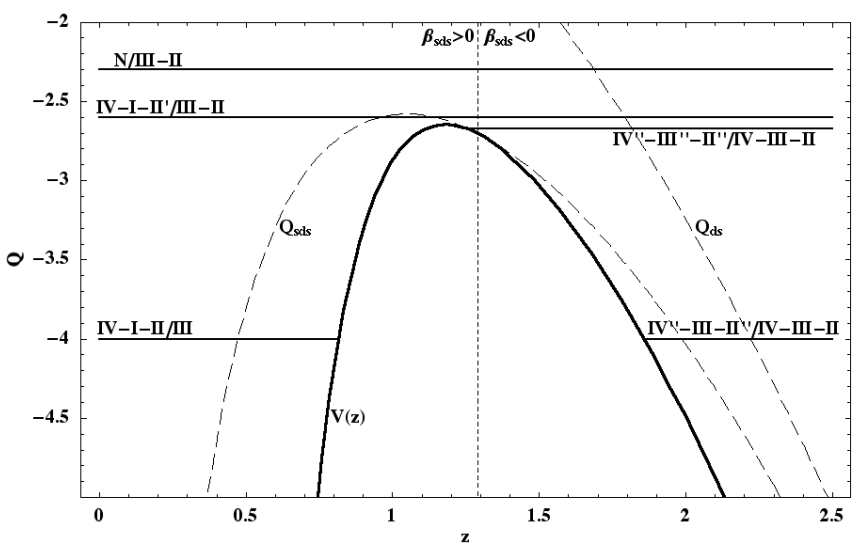

FIG. 8: Potential for $A=2.9$ and $B=3$. For this choice of parameters, the sign change in $\beta_{\text {sds }}$ occurs to the right of the maximum in the potential and there is no $\beta_{\mathrm{ds}}$ sign change. Various trajectories are noted.

$\left.\Lambda_{+}<\Lambda_{-}\right)$and $M \geq 0$ are denoted in Fig. 5 , 6 , and 8 Figures 9 and 10 display the conformal structure of these solutions [39]. The conformal diagrams in each row are matched along the bubble wall (solid line with an arrow) and the physical regions are shaded. For solutions with qualitatively similar SdS diagrams, the various options for the dS interior are listed. The naming scheme in Fig. [5 6 7 and 8 is chosen to reflect the structure of the conformal diagrams. The first numbers are the regions of the SdS conformal diagram that the bubble wall passes through. The numbers following the backslash are the regions of the $\mathrm{dS}$ conformal diagram that the bubble wall passes through.

For example, consider the IV-I-II/III solution (solution 1 of Fig. (9). These start at at $r=z=0$, corresponding to the singularity in region IV of the SdS conformal diagram and the $r=0$ surface in region III of the dS conformal diagram. $\beta_{\mathrm{sds}}$ and $\beta_{\mathrm{ds}}$ are both greater than zero over the entire trajectory. Therefore, the wall on the SdS side must follow a path of increasing $t_{\text {sds }}$, pushing it into region I (crossing the black hole horizon). The wall on the dS half must follow a path of decreasing coordinate time, and thus remain in region III. The wall then reaches a turning point, falls through the black hole event horizon, and ends up back at $r=z=0$ (the singularity in region II of the SdS diagram, and the $r=0$ surface in region III of the dS diagram). The construction of the other diagrams in Fig. 9 and Fig. 10 proceeds similarly.

Based on the inward pressure gradient, the IV-I-II/III solution is what one might expect the junction conditions to yield: a sphere of false vacuum which expands and then contracts. Relativistic effects, however, lead to the qualitatively different behavior exhibited by the other solutions in Fig. 9 and Fig. 10 For instance, the evolution of the IV-III-II/III (solution 2) solution is qualitatively similar to the IV-I-II/III solution, but is so massive that its evolution is always hidden behind the black hole event horizon. The IV-I-II'/III-II (solutions 3 and 4) solution 


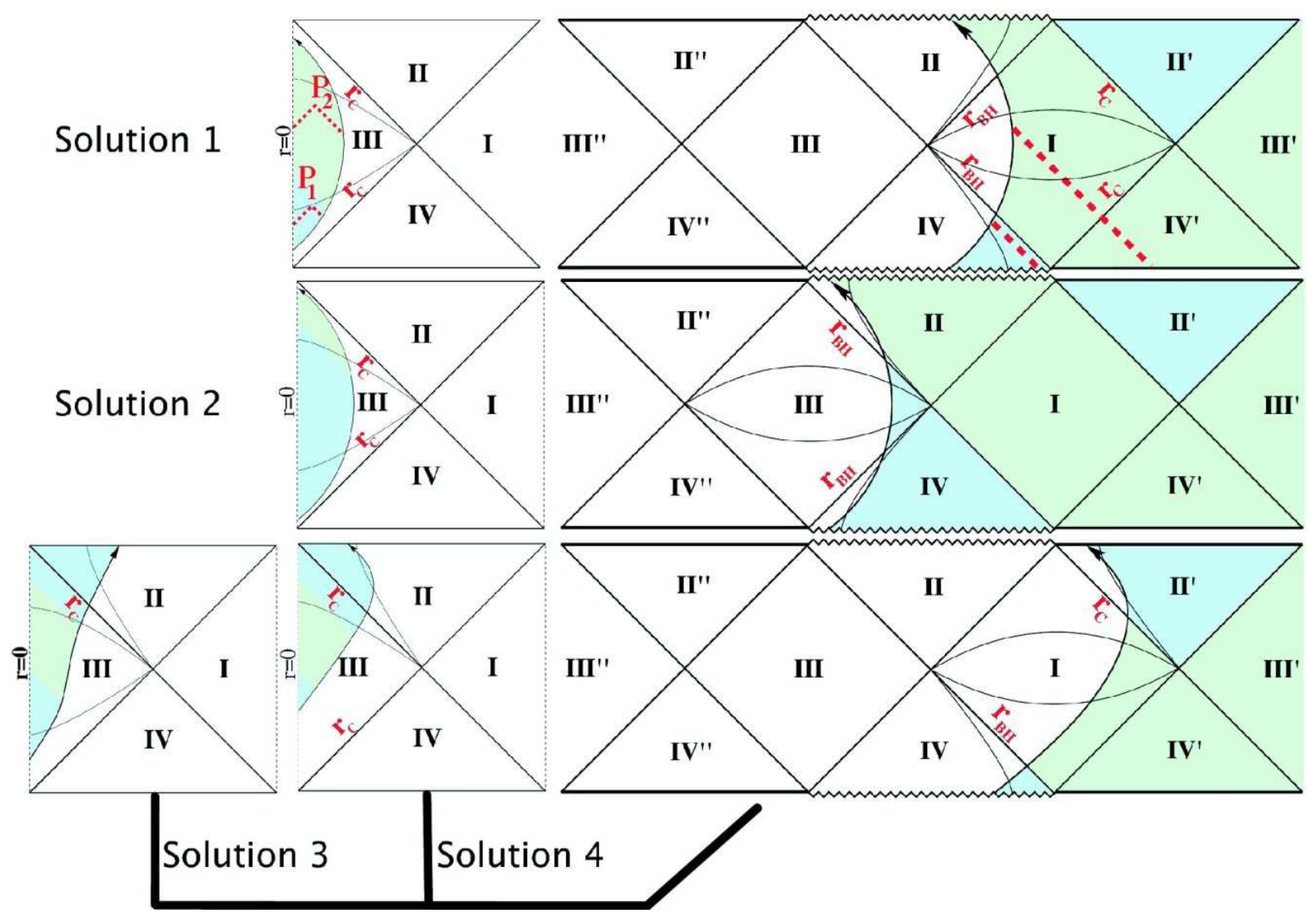

FIG. 9: Conformal diagrams for the allowed one-bubble spacetimes. The dS and SdS diagrams are matched across the bubble wall (line with arrow), and the physical regions shaded. Solutions 3 and 4 share the same SdS diagram. Regions which do not contain anti-trapped surfaces are shaded green (light), regions which do are shaded blue (dark).

is a bubble which has enough kinetic energy to escape collapse by expanding through the cosmological horizon; observers inside (or who travel inside from region I) the false vacuum region will find themselves in an inflationary universe at late times. In the time-reverse of this solution, a bubble implodes from infinity into the black hole horizon, and the interior undergoes collapse.

Note that there are two options for the IV-I-II'/IIIII solution. Solution 3 corresponds to the situation $A+3<B<3(A-1)$, which contains both $\beta_{\text {sds }}$ and $\beta_{\mathrm{ds}}$ sign changes (see Fig. 5). This causes the bubble wall to accelerate in the direction of the true vacuum from both the interior and exterior perspectives. Solution 4 corresponds to the situation $B<A+3$, which does not contain a $\beta_{\mathrm{ds}}$ sign change (see Fig. 8). This causes the bubble wall to accelerate in the direction of the false vacuum from the interior perspective. The reason for the disparity is that solution 4 exists only when the interior and exterior cosmological constants are similar in magnitude, and so the wall tension can have greater influence on the dynamics.

The remaining solutions, shown in Fig. 10, have interiors which approach an inflationary universe at late times, but lie on the opposite side of the wormhole in the exterior SdS spacetime. To an observer in region III of the SdS diagram, the IV"-III-II" /IV-I-II (solutions 5) and IV"-III-II" /IV-III-II solutions (solutions 6 and 7) would appear as a "sky" of false vacuum that encroaches from infinity, reaches a minimum radius and then expands back out. It has been pointed out by Bousso [13] that at late times (in an asymptotically flat spacetime) the wall trajectory according to the observer in region III of the $\mathrm{S}(\mathrm{dS})$ diagram approaches that of a true vacuum bubble [21, 22, 23]. Amusingly, the SdS observer will think he is in a true vacuum bubble surrounded by a large region of false vacuum, while the dS observer will think she is in a false vacuum bubble surrounded by a large region of true vacuum.

This symmetry between true and false vacuum bubbles is made manifest in the analysis of the Coleman-De Luccia 23] instanton, which describes the production of both true and false vacuum bubbles 10. These are zero energy solutions, and so we should look for an $M=0$ unbound solution; this corresponds (via Eq. 201) to $Q \rightarrow-\infty$, and we see from Fig. 15 6, 7 and 8 that the IV"-III-II" /IV-I-II solution (solution 5) or the IV"-III-II" /IV-III-II solution (solution 6), depending on the values of $\Lambda_{+}$and $\Lambda_{-}$, can be identified as the analytically continued false vacuum 


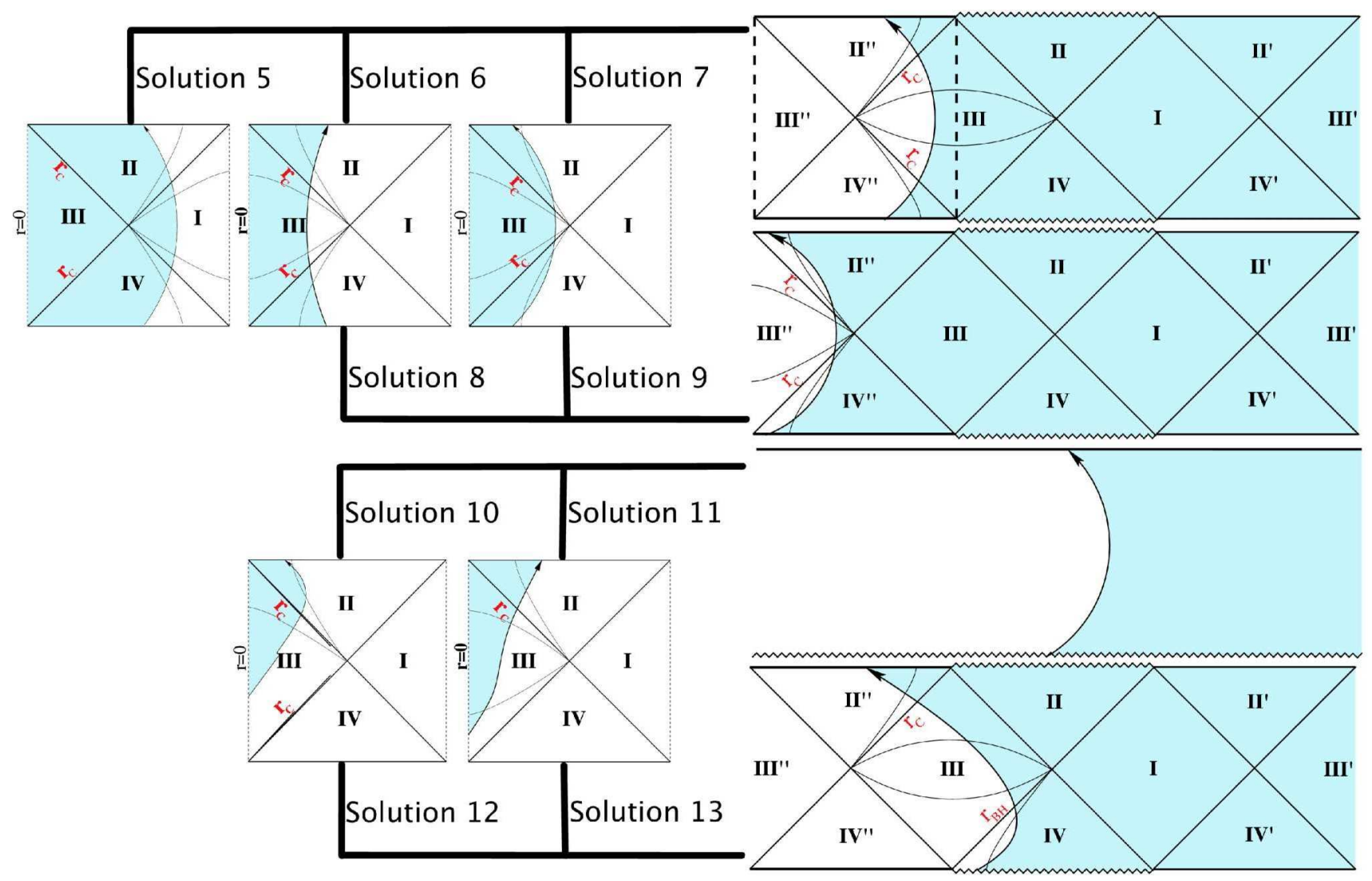

FIG. 10: Conformal diagrams for the allowed one-bubble spacetimes. The dS and SdS diagrams are matched across the bubble wall (line with arrow), and the physical regions shaded. For solutions with qualitatively similar SdS diagrams, the various options for the dS interior are listed.

instanton. The radius of the bubble wall at the turning point is found by considering the limit as the potential (Eq. 18) goes to $-\infty$, where on the right (unbound) side of the potential hump the $z^{2}$ term dominates. Solving for $r$ using Eq. 14, we find the radius at turnaround to be

$$
r=6 k\left[\left|\left(\Lambda_{+}+\Lambda_{-}+3 k^{2}\right)^{2}-4 \Lambda_{+} \Lambda_{-}\right|\right]^{-1 / 2}
$$

which agrees with the previous literature [16] (see also [11]). Since the Schwarzschild mass is zero, we are now matching two pure de Sitter spacetimes across the bubble wall. The conformal diagram for the exterior dS region (right) only contains the area between the vertical dashed lines (which are now identified as $r=0$ surfaces) in solution 5 and 6 of Fig. 10] The interior half (left) of the diagram remains unchanged. It can be seen that at turnaround, the bubble will be larger than both the interior horizon size and the (exterior) horizon size of the region it has replaced.

In the IV-III-II" /III-II solution (solutions 12 and 13), the region of false vacuum surrounding the observer in region III of the SdS diagram would begin very small and then expand out of the cosmological horizon. This solution will also have a time-reversed version in which the surrounding region of false vacuum implodes. For the IV"-III"-II"/IV-III-II solution (solutions 8 and 9), the "sky" of false vacuum would forever reside outside of the horizon of a region III observer. Finally, the N/III-II solution (solutions 10 and 11), which we will define as solutions with mass greater than or equal to the Nariai mass, will be an exploding (or imploding in the timereversed solution) bubble of false vacuum centered on $r=0$.

In a series of papers, Farhi et. al. 1, 2] discussed the application of the Penrose theorem 24] to the one-bubble spacetimes discussed above. If the null energy condition (NEC) holds (as it does for the postulated energy momentum tensor) and there exists a non-compact Cauchy surface (as in the full SdS spacetime), then the existence of a closed anti-trapped surface in the spacetime implies the presence of an initial singularity. Since each point on the conformal diagrams in Fig. 9 and Fig. 10 represents a two-sphere, such an anti-trapped surface exists if the ingoing and outgoing future-directed null rays both diverge. For example, the 2 -sphere represented by point $P_{1}$ shown in Fig. 9] solution 1, is a closed antitrapped surface. This can be seen by following the null ray (null rays are denoted by the dashed lines in Fig. (9) from $r=0$ in region III of the dS diagram to $P_{1}$ and not- 
ing that $r$ increases monotonically as $P_{1}$ is approached (the null rays are diverging). But following the futuredirected null rays in the opposite direction from $r=0$ in region IV of the SdS diagram, across the bubble wall, and into the false vacuum region, shows that they also diverge. Thus, an initial singularity is necessary for this solution to exist at and near $P_{1}$. This spacetime also, however, contains regions without anti-trapped surfaces. Following the future-directed null rays to point $P_{2}$, for example, we see that the ingoing rays diverge, but the outgoing rays converge. For examples, see solutions 1-4 in Fig. 9] where regions which contain anti-trapped surfaces are shaded blue (dark) and the regions which do not are shaded green (light).

If we cut the IV-I-II/III solution (solution 1) in the expanding phase on a spacelike hypersurface at a time where the radius of the bubble wall satisfies $r>r_{B H}$, then the spacetime would not necessarily contain an initial singularity [40]. We can remove the initial singularity from the IV-I-II'/III-II (solution 3 and 4) solution as well by cutting on the same surface. Both the IV-I-II/III (solution 1) and IV-I-II'/III-II (solution 3 and 4) solutions are therefore classically buildable. The IV-I-II'/III-II solution (solutions 3 and 4 ) is the only example where it is possible to form an inflationary universe from classically buildable initial conditions, but only exists when the interior and exterior cosmological constant are almost equal $(B<3(A-1))$. This solution might be of interest in understanding transitions between nearly degenerate vacua, for example in the context of eternal inflation.

Given the existence of a classically forbidden region in Fig. 5] 6] 7. and 8, one might ask if tunneling is allowed from one of the recollapsing solutions (solutions 1 and 2) to one of the expanding solutions (solutions 5-9 ) on the other side of the potential hump. This event, shown in Fig. 11] would constitute a violation of the NEC, and so the Penrose theorem would no longer apply to the antitrapped surfaces that exist after the tunneling event. Such a process is indeed apparently allowed [2, 3], and would describe the quantum creation of an inflationary universe from classically buildable initial conditions (if the initial condition is the IV-I-II/III solution in solution $1)$.

This is a rather strange transition, however, as the unbound solution is behind the wormhole in SdS (see Fig. 11). An observer in region I would see the bubble expand, reach its turning point, and then disappear, only to be replaced by a black hole. An observer inside the bubble would see the wall expanding away and - just as it is about to turn around and start collapsing - instead disappear behind the cosmological horizon. This observer will be inside an inflationary universe, but forever disconnected from region I. If the black hole in the SdS spacetime then evaporates, the baby universe will become completely topologically disconnected.

Having considered one circumstance in which an NEC violation precipitates the creation of an inflationary universe, one might ask if there are others. Quantum fluc-

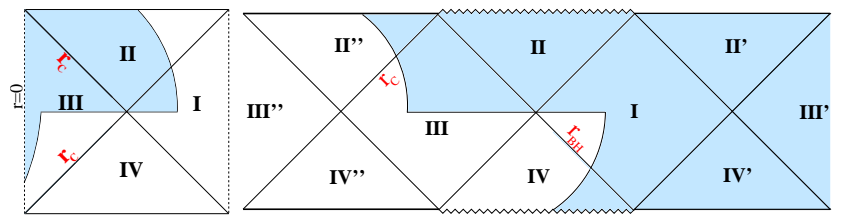

FIG. 11: Tunneling spacetime

tuations of a scalar field in de Sitter space can violate the NEC, and so one might imagine that any of the solutions that we have discussed which allow inflation inside the bubble could be spontaneously created somewhere along their trajectories. One example of this direct production of baby universes is a fluctuation into one of the unbound solutions (solutions 5-9). Such scenarios have been considered in the context of the stochastic approach to baby universe production by Linde [25] and in reference to eternal inflation by Carroll and Chen 7].

Another example of the direct fluctuation into an inflationary universe is the thermal decay of de Sitter vacua discussed by Garriga and Megevand [26] (Ref. 27] discusses a related mechanism). This process consists of the fluctuation from empty de Sitter of a bubble in unstable equilibrium between expansion and collapse. This static solution is identified as the set of spacetimes which sit on top of the potentials in Fig. 5] 6] 7. and 8] The two possible configurations are shown in Fig. 12] where it can be seen that the bubble wall can lie on either side of the worm hole depending on the sign of $\beta_{\text {sds }}$ at the top of the potential. It can be shown that the "Nariai limit" in Ref. 26] corresponds to $B=3(A-1)$, where the $\beta_{\text {sds }}$ sign change occurs at the max of the potential.
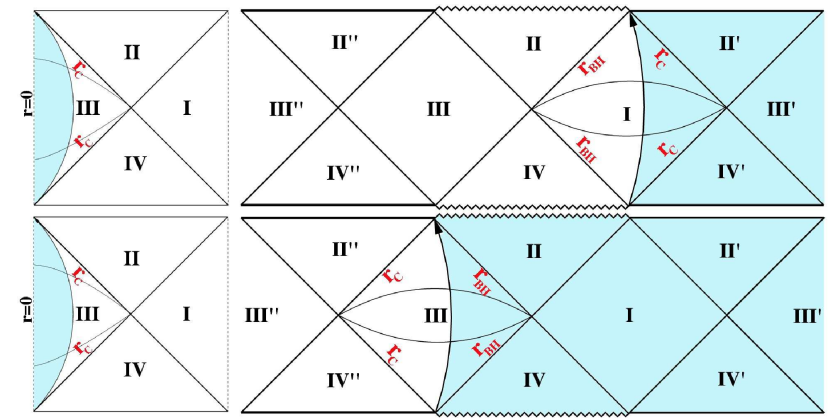

FIG. 12: Conformal diagrams for the thermal decay of de Sitter vacua. The upper diagram corresponds to $B<3(A-1)$, the lower diagram corresponds to $B>3(A-1)$.

With these considerations, there are at least three competing channels for the formation of baby universes: direct production, the FGG mechanism, and the zero-mass false vacuum instanton. It would be desirable to develop a scheme to directly compare the relative probabilities of each of these processes, and this is currently being explored.

Our full catalog of solutions is also interesting in regards to a recent proposal [14, 15] that false vacuum re- 
gions, assumed to be larger than the interior horizon, must at all times be larger than the exterior, true vacuum, horizon. The basis of this conjecture is the condition that the divergence of a congruence of future directed null geodesics (defined as $\theta$ ) must satisfy

$$
\frac{d \theta}{d T} \leq 0
$$

where $T$ is an affine parameter, if the NEC holds for all $T$. Null rays in the dS and SdS spacetimes satisfy this inequality (in $\mathrm{dS}$, the inequality is exactly zero), but we should check that the junction conditions do not violate it. One requirement imposed by Eq. 23$]$ is that the divergence of the null rays does not increase at the position of the wall as they go from a true vacuum region into a false vacuum one. Along any given null geodesic in the bubble interior or exterior, the value of $r$ is either increasing or decreasing monotonically as a function of $T$. We can therefore state the condition Eq. 23$]$ as: one cannot have a null ray along which $d r / d T \leq 0$ outside the bubble and $d r / d T \geq 0$ inside the bubble. Surveying the solutions in Fig. 9 and Fig. 10] we see that this is indeed always true.

The authors of Ref. 14, 15] intended to demonstrate that if one requires the false vacuum region to be larger than the interior horizon size at all times (so that inflation is unstoppable), it is necessarily larger than the exterior horizon size. Although all of the allowed onebubble spacetimes satisfy the condition Eq. 23] there are only two examples in which all observers agree that this requirement is met: the false vacuum instanton (IV"III-II" /IV-I-II solution (solution 5), with $M=0$ ) and the IV-I-II'/III-II solution (solution 3 and 4, after turnaround). In every other case (including the FGG spacetime in Fig. [11), the observers in region I of the SdS conformal diagram will see only a black hole horizon sized volume replaced by the false vacuum bubble. We therefore conjecture that if one requires the false vacuum region to be larger than the interior horizon size at all times, then it will replace a volume larger than the exterior horizon size according to only some observers. If one relaxes this requirement, then there are a diverse range of solutions which might describe the spawning of an inflationary universe. For example, the bubbles in solutions 3, 4 and 10-13 of Fig. 9 and 10 grow from an arbitrarily small size.

Having discussed the character of the various solutions, we turn now to a potentially dangerous detail, which is particularly important for the FGG mechanism: there exists a classical instability against aspherical perturbations in the spherically symmetric solutions to the junction conditions.

\section{PERTURBATIONS}

The solutions described in Sec. IIC assume that the region of false vacuum is spherically symmetric. The stability of these solutions against aspherical perturbations has important consequences, especially if one hopes to build plausible cosmologies. That there might be an instability in domain walls was first discussed by Adams, Freese and Widrow 28]. The bubble wall can trade volume energy for surface energy and wall kinetic energy locally as well as globally, and so the bubble wall will become distorted if different sections of the wall have different kinetic energies. As long as the local distortions of the wall remain small compared to the size of the background solution's radius, this process can be formulated quantitatively as perturbation theory around a background spherically symmetric solution.

Previous authors [28, 29, 30] have considered perturbations on expanding bubbles of true-vacuum [21, 22, 23], which have zero total energy (surface, volume, and kinetic energies canceling), and so can expand asymptotically. As was first pointed out by Garriga and Vilenkin [29], even though local observers on the bubble wall see perturbations grow, external observers see them freeze out because they do not grow faster than bubble radius.

The story is different for the bound (solutions 1 and 2 ) and unbound (solutions 5-9) false vacuum bubbles: since they reach a turning point, the perturbations have a chance to catch up to the bubble's expansion and become nonlinear. This also presumably has implications for the thermal decay mechanism of Garriga and Megevand [26], depending on the duration of time the bubble wall sits in unstable equilibrium between expansion and collapse (see discussion in Sec. IC). The remainder of this work will focus on the instability of the bound IV-I-II/III solution (solution 1), since physically plausible initial conditions may be clearly formulated. There is no obvious set of initial conditions for the perturbations on the unbound solutions, and so we simply observe that the results we will obtain for the bound solutions apply qualitatively here as well.

To simplify the problem, we assume that the full gravitational problem described in the previous sections can be treated as motion of the bubble wall in a fixed SdS background. This assumption must be validated (as we do below), but we are mainly interested in the low-mass bound solutions for which we might expect the gravitational contributions to be small. Assuming that a thin spherically symmetric bubble wall separates an internal dS from an external SdS spacetime, we can employ the action [28, 29, 30]:

$$
S=-\sigma \int d^{3} \xi \sqrt{-\gamma}+\epsilon \int d^{4} x \sqrt{-g},
$$

where $\sigma$ is the surface energy density on the bubble wall, $\gamma_{a b}(a, b=1,2,3)$ is the metric on the worldsheet of the bubble wall, $\epsilon$ is the difference in volume energy density on either side of the bubble wall:

$$
\epsilon=\frac{\Lambda_{+}-\Lambda_{-}}{8 \pi}
$$

and $g_{\alpha \beta}$ is the metric of the background spacetime. 


\section{A. Wall Equation of Motion}

The equation of motion resulting from Eq. 24] is [30]:

$$
g^{a b} K_{a b}=-\frac{\epsilon}{\sigma}
$$

where $K_{a b}$ is the extrinsic curvature tensor of the worldsheet of the bubble wall,

$$
K_{a b}=-\partial_{a} x^{\mu} \partial_{b} x^{\nu} D_{\nu} n_{\mu}
$$

and $D_{\nu}$ is the covariant derivative and $n_{\mu}$ is the unit normal to the bubble wall worldsheet.

We will use the static foliation of the SdS spacetime (see Eq. 1) as the coordinates $x^{\mu}$ for the background spacetime. The world sheet is given coordinates $(\tau, \theta, \phi)$ as in Eq. 7, and has metric:

$$
\gamma_{a b}=g_{\mu \nu} \partial_{a} x^{\mu} \partial_{b} x^{\nu}
$$

with the gauge freedom in choosing $\tau$ fixed by

$$
\frac{d t}{d \tau} \equiv t^{\prime}=\frac{\sqrt{a+r^{\prime 2}}}{a},
$$

so that $\gamma_{\tau \tau}=-1$. Here and henceforth primes will denote derivatives with respect to $\tau$. The other non-zero components of $\gamma_{a b}$ are $\gamma_{\theta \theta}=r^{2}$ and $\gamma_{\phi \phi}=r^{2} \sin ^{2} \theta$.

The first task at hand is to find the worldsheet's unit normal, which by spherical symmetry has only $r$ and $t$ components. Requiring orthogonality to the worldsheet $\left(g_{\mu \nu} n^{\nu} \partial_{a} x^{\mu}=0\right)$ and unit norm $\left(g_{\mu \nu} n^{\mu} n^{\nu}=1\right)$ yields its components:

$$
n_{t}=-r^{\prime}, \quad n_{r}=t^{\prime}
$$

The components of $K_{a b}$ are given by

$$
\begin{aligned}
& K_{\tau \tau}=\left[r^{\prime \prime}+\frac{1}{2} \frac{d a}{d r}\right]\left(a+r^{2}\right)^{-1 / 2}, \\
& K_{\phi \phi}=-r a t^{\prime} \sin ^{2} \theta=K_{\theta \theta} \sin ^{2} \theta .
\end{aligned}
$$

Substituting Eq. 31] into Eq. 26 gives the equation of motion for the bubble wall:

$$
r^{\prime \prime}=\frac{\epsilon}{\sigma} \sqrt{a+r^{\prime 2}}-\frac{2}{r}\left(a+r^{2}\right)-\frac{1}{2} \frac{d a}{d r} .
$$

Eq. 18] supplies the velocity of the bubble at some position along its trajectory

$$
z^{\prime}=\left[Q-V\left(z_{0}\right)\right]^{1 / 2} .
$$

Choosing this boundary condition is effectively restricting ourselves to the IV-I-II/III (solution 1) or IV-IIIII/III (solution 2) solutions. Since the solutions to Eq. 32 approximate the dynamics of the junction condition problem, we should parametrize by $A, B$, and $Q$.
This can be done by using the conversions defined in Sec. IIB and gives:

$$
\begin{aligned}
z^{\prime \prime} & =-\frac{3(B-A)}{c} \sqrt{a(-Q)+z^{\prime 2}} \\
& -\frac{2}{z}\left(a(-Q)+z^{\prime 2}\right)-\frac{(-Q)}{2} \frac{d a}{d z}
\end{aligned}
$$

where $a$ is written in terms of $z$ as

$$
a=1-\frac{12}{c z(-Q)}-\frac{12 A}{c^{2}(-Q)} z^{2},
$$

and

$$
c \equiv\left[\left|(A+B+3)^{2}-4 A B\right|\right]^{\frac{1}{2}} .
$$

To justify the use of the simplified dynamics described above, Eq. 34 was numerically integrated, and the position of the turning point compared to the corresponding point on the full junction condition potential. Over the range of $Q$ corresponding to the bound solutions, we find excellent quantitative agreement (well within 1\%) between the turning points of the solutions to Eq. 34 and the junction condition potential. This was repeated with equally good results for the weak, GUT, and Planck scale potentials and also for various initial positions between the black hole radius and the potential wall (turning point). This shows that to zeroth order, dynamics as motion in a background is valid, and strongly suggests that it will be at higher orders as well.

\section{B. Perturbations}

We are now in a position to discuss the first-order perturbations on the spherically-symmetric background solutions discussed in Sec. IIIA Physical perturbations are normal to the worldsheet of the (background) bubble wall, and can be described by scalar field $\phi(x)$ by taking the position of the perturbed worldsheet to be

$$
\bar{x}^{\mu}=x^{\mu}+\phi(x) n^{\mu},
$$

where $x^{\mu}$ is the spherically symmetric solution, and $n^{\mu}$ is the unit normal to the worldsheet. It is assumed that $\phi$ is much smaller than the bubble wall radius, so that a perturbative analysis can be made.

The equation of motion for the perturbation field $\phi(x)$ in a curved spacetime background can be derived from the action Eq. 24 after expanding to second order in $\phi(x)$ 30 ]

$$
\triangle \phi-\left[-R_{\mu \nu} h^{\mu \nu}+R^{(3)}-\frac{\epsilon^{2}}{\sigma^{2}}\right] \phi=0,
$$

where

$$
\triangle \phi=\frac{1}{\sqrt{-\gamma}} \partial_{a}\left(\sqrt{-\gamma} \gamma^{a b} \partial_{b} \phi\right)
$$


and $h^{\mu \nu}$ is

$$
h^{\mu \nu}=g^{\mu \nu}-n^{\mu} n^{\nu}
$$

To solve the equation of motion, we can decompose $\phi(x)$ into spherical harmonics

$$
\phi(x)=\sum_{l, m} \phi_{l m}(\tau) Y_{l m}(\theta, \phi)
$$

and separate variables to get an equation for $\phi_{l m}(\tau)$. The geometrical factors in Eq. 38 become dependent on $\theta$ or $\phi$ only at second order, so we will always be able to make this decomposition.

$\triangle \phi$ is then given by:

$$
\Delta \phi_{l m}=-\left(\partial_{\tau}^{2}+\frac{2 r^{\prime}}{r} \partial_{\tau}+\frac{l(l+1)}{r^{2}}\right) \phi_{l m} .
$$

The components of $h^{\mu \nu}$ are:

$$
\begin{aligned}
& h^{t t}=-\frac{a+r^{\prime 2}}{a^{2}}, \quad h^{r r}=-r^{\prime 2}, \\
& h^{\theta \theta}=h^{\phi \phi} \sin ^{2} \theta=\frac{1}{r^{2}} .
\end{aligned}
$$

The components of the Ricci tensor are given by:

$$
R_{t t}=\frac{a}{2} \partial_{r}^{2} a+\frac{a}{r} \partial_{r} a=-a^{2} R_{r r}
$$

$$
R_{\phi \phi}=R_{\theta \theta} \sin ^{2} \theta=\left(1-a-r \partial_{r} a\right) .
$$

Contracting equations 43 and 44 gives:

$$
R_{\mu \nu} h^{\mu \nu}=\frac{2(1-a)}{r^{2}}-\frac{3 \partial_{r} a}{r}-\frac{\partial_{r}^{2} a}{2}=3 \Lambda_{+} .
$$

The Ricci scalar on the world sheet is

$$
R^{(3)}=\frac{2}{r^{2}}\left(1+r^{\prime 2}+2 r r^{\prime \prime}\right)
$$

where $r^{\prime \prime}$ is given by Eq. 32

After substituting Eq. 42 Eq. 45, and Eq. 46 into Eq. 38. the equation of motion for $\phi_{l m}(\tau)$ is

$$
\phi_{l m}^{\prime \prime}=\left[\frac{\epsilon^{2}}{\sigma^{2}}-\frac{4 \epsilon}{\sigma r}\left(a+r^{\prime 2}\right)^{1 / 2}+3 \Lambda_{+}+\frac{2}{r} \frac{d a}{d r}+\frac{6 r^{\prime 2}}{r^{2}}-\frac{2(1-4 a)}{r^{2}}-\frac{l(l+1)}{r^{2}}\right] \phi_{l m}-\frac{2 r^{\prime} \phi_{l m^{\prime}}}{r} .
$$

In terms of the dimensionless variables of the junction condition problem this reads:

$$
\begin{aligned}
& \Phi_{l m}^{\prime \prime}=-\frac{2 z^{\prime}}{z} \Phi_{l m}^{\prime} \\
& +\left\{\frac{108 A}{c^{2}}+\frac{9(A-B)^{2}}{c^{2}}+\frac{12(B-A)}{c z}\left(a(-Q)+z^{\prime 2}\right)^{1 / 2}+\frac{2(-Q)}{z^{2}}(4 a-1)+\frac{6 z^{\prime 2}}{z^{2}}+\frac{2(-Q)}{z} \frac{d a}{d z}-\frac{l(l+1)(-Q)}{z^{2}}\right\} \Phi_{l m}
\end{aligned}
$$

where $\Phi$ is the dimensionless perturbation field defined similarly to $z$ (see Eq. 14). The first term acts as a (anti)drag on (shrinking) growing perturbations. The last term in this equation is always negative, acting as a restoring force. Perturbations will grow when the other terms (which are positive over most of the trajectory in the expanding phase) in this equation dominate. Further, the last term indicates that lower $l$ modes will experience the largest growth. The full details of the solutions, however, require a numerical approach, to which we now turn.

\section{APPLICATION TO THE FARHI-GUTH-GUVEN MECHANISM}

The possibility of creating an inflating false-vacuum region via quantum tunneling (described in Sec. IC) has been investigated only under the assumption of spherical symmetry, and this would be grossly violated if perturbations on the bubble wall become nonlinear. In this section, we investigate the circumstances under which this is the case. The two basic questions at issue are: first, when do perturbations go nonlinear for some given set of initial perturbations, and second, what initial perturbations can be expected.

\section{A. Dynamics of the Perturbation Field}

Let us begin with the first issue. Since Eq. 48 is a second order ODE, it can be decomposed into the sum of two linearly independent solutions

$$
\begin{aligned}
\Phi_{\operatorname{lm}}(T)= & \Phi_{\operatorname{lm}}(T=0) f\left(l, z_{0}, Q, T\right) \\
& +\Phi_{\operatorname{lm}}^{\prime}(T=0) g\left(l, z_{0}, Q, T\right) .
\end{aligned}
$$


The functions $f\left(l, z_{0}, Q, T\right)$ and $g\left(l, z_{0}, Q, T\right)$ can be found numerically by alternately setting $\Phi_{\operatorname{lm}}(T=0)$ and $\Phi_{\operatorname{lm}}^{\prime}(T=0)$ to zero, then evolving the coupled Eq.48] and Eq. 34 numerically for a time $T$ with initial conditions for $Q, z_{0}$, and $l$. If the bubble is to tunnel, it will do so at the time $T_{\max }$, when the bubble reaches its maximum radius and begins to re-collapse. Given $f$ and $g$ at time $T_{\max }$, the size of the perturbations at the turning point for any $z_{0}, Q, l, \Phi_{\operatorname{lm}}(T=0)$, and $\Phi_{\operatorname{lm}}^{\prime}(T=0)$ can be determined. An RK4 algorithm with adaptive step size was used to solve for $f$ and $g$, with numerical errors well within the $1 \%$ level.

The results of this analysis for $l=1$ and for the low (weak) and intermediate (GUT) inflation scales discussed below Eq. 21] are shown in Fig. 13] The solid lines show contours of constant (log) amplification factor $f$ (left) and $g$ (right) versus the bubble starting radius $z_{0}$ and mass parameter $Q$, with bubble mass increasing toward the top. The shaded regions indicate regions which we have disallowed as bubble starting radii because the bubble would not be classically buildable for $r<r_{\mathrm{BH}}$ (marked as $Q>Q_{\mathrm{BH}}$ ), or the bubble is in the forbidden region $Q<V(z)$ of the effective 1D equation of motion Eq. 17. or the bubble would be too small to be treated classically. We choose the latter radius as fifty times the Compton wavelength $z_{\text {compton }}$ of a piece of the bubble wall [41]. The choice of fifty Compton wavelengths is rather arbitrary; the effect of a larger bound would be to exclude more of the parameter space in Fig. 13. This (unshaded) parameter space includes all classical initial conditions which could be set up by the observer in region I of the SdS conformal diagram.

It can be seen in Fig. 13 that the growth of the perturbations is in general larger for higher-mass bubbles (smaller $|Q|$, larger $-\log _{10}(-Q)$ ). The lower the inflation scale, the closer to zero the peak in the potential function becomes, and the smaller $|Q|$ (higher mass) bubbles are allowed, so at low inflation scales $f$ and $g$ can be very large. Growth for the Planck-scale inflation bubbles is very small, with $f$ of order 10 and $g$ of order 1 , and is not plotted.

The enhanced growth at small $|Q|$ is due to the suppression of the term in Eq. 48 proportional to $l(l+$ $1)(-Q)$, which always acts to stabilize the perturbations. Another consequence of this suppression is that the range in $l$ over which solutions are unstable depends on $Q$; as a general rule of thumb, approximately a few times $(-Q)^{-1 / 2} l$-modes are unstable (note that this is unlike the case true vacuum bubbles, for which only the $l=0,1$ modes are unstable). An example of the $f$ function for $Q=-10^{-4}$ with the intermediate (GUT) inflation scale parameters is shown in Fig. 14. The $f$ functions for very large $l$ modes are stable and approach sinusoids with amplitudes less than one (see the inset of Fig. 14), meaning that the perturbations are never larger than their initial size.

\section{B. Initial Conditions and Evolution to the Turning Point}

Having fully characterized the growth of the perturbations, we now require an estimate for their initial values when the bubble is formed. There is no reason to expect that a region of false vacuum will fluctuate into existence with anything near spherical symmetry, nor is it likely to have thin walls (there is no instanton or other mechanism to enforce these symmetries). Since low- $l$ (relative to $(-Q)^{-1 / 2}$ modes are unstable, an initially aspherical bubble will only become more aspherical; this is in marked contrast to true vacuum bubbles, which both start spherical, and tend to become more spherical as they expand.

Suppose, however, that we consider the best-case scenario in which a bubble is, by chance or design, spherically symmetric. It will nevertheless inevitably be dressed with zero-point quantum fluctuations of the perturbation field. We may then check whether these fluctuations alone, considered as initial values for the perturbations of a bubble starting with a given $Q$ and $z_{0}$, suffice to make the bubble nonlinearly aspherical by turnaround.

We assume that the ensemble average of the quantum fluctuations at the time of nucleation is zero; but the ensemble average of the square of the field (the space-like two-point function $\langle\Phi(\theta, \phi) \Phi(\tilde{\theta}, \tilde{\phi})\rangle \equiv\langle\Phi \tilde{\Phi}\rangle)$ will not generally vanish. We can write the mode functions (Eq. 41) in terms of it as:

$$
\left\langle\Phi_{\operatorname{lm}}^{2}\right\rangle=\begin{aligned}
& \int d \Omega d \tilde{\Omega}\langle\Phi \tilde{\Phi}\rangle Y_{\operatorname{lm}}(\theta, \phi) Y_{\operatorname{lm}}^{*}(\tilde{\theta}, \tilde{\phi}) .
\end{aligned}
$$

By spherical symmetry, the two-point function can be written as a function of the angular separation $\Psi$ between $(\theta, \phi)$ and $(\tilde{\theta}, \tilde{\phi})$, and decomposed into Legendre polynomials:

$$
\langle\Phi \tilde{\Phi}\rangle=\sum_{l} C_{l} P_{l}(\cos \Psi) .
$$

Using the addition theorem for spherical harmonics, we can write this as

$$
\langle\Phi \tilde{\Phi}\rangle=\sum_{\mathrm{l}^{\prime}, \mathrm{m}^{\prime}} \frac{4 \pi}{2 l^{\prime}+1} C_{l^{\prime}} Y_{\mathrm{l}^{\prime} \mathrm{m}^{\prime}}^{*}(\theta, \phi) Y_{\mathrm{l}^{\prime} \mathrm{m}^{\prime}}(\tilde{\theta}, \tilde{\phi}) .
$$

Substituting this into Eq. [50] and using the orthogonality of the spherical harmonics yields the relation:

$$
\left\langle\Phi_{\operatorname{lm}}^{2}\right\rangle=\frac{4 \pi C_{l}}{2 l+1} .
$$

Given some space-like two point function at the time the bubble is nucleated, we can obtain the $C_{l}$ from

$$
C_{l}=\frac{2 l+1}{4 \pi} \int_{-1}^{1} d(\cos \Psi)\langle\Phi \tilde{\Phi}\rangle P_{l}(\cos \Psi)
$$



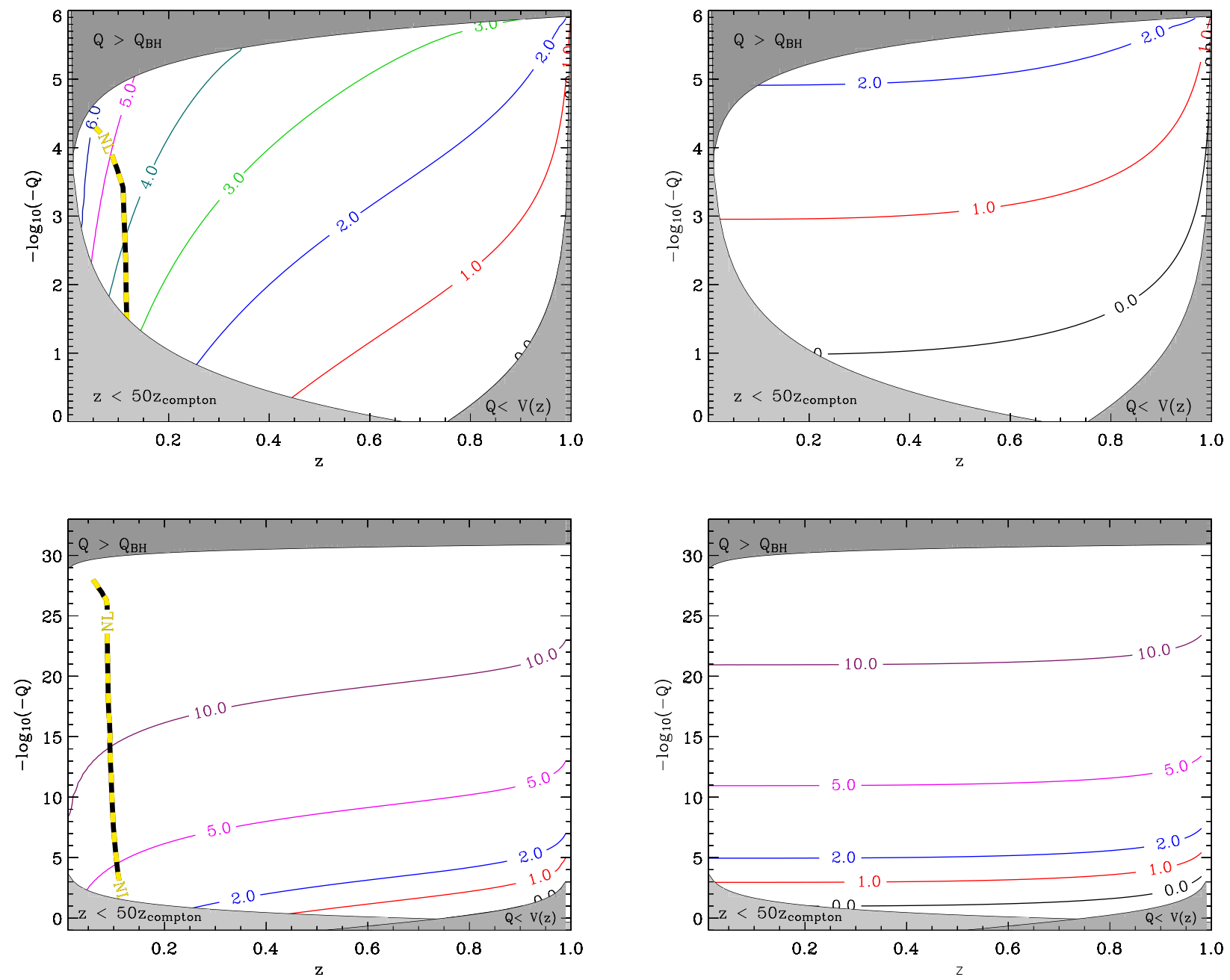

FIG. 13: (Color online) Contour plot of $\log _{10}\left[f\left(l=1, z_{0}, Q, T_{\max }\right)\right]\left(\right.$ left) and $\log _{10}\left[g\left(l=1, z_{0}, Q, T_{\max }\right)\right]$ (right) for $M_{I}=10^{14}$ $\mathrm{GeV}$ (top) and $M_{I}=100 \mathrm{GeV}$ (bottom).

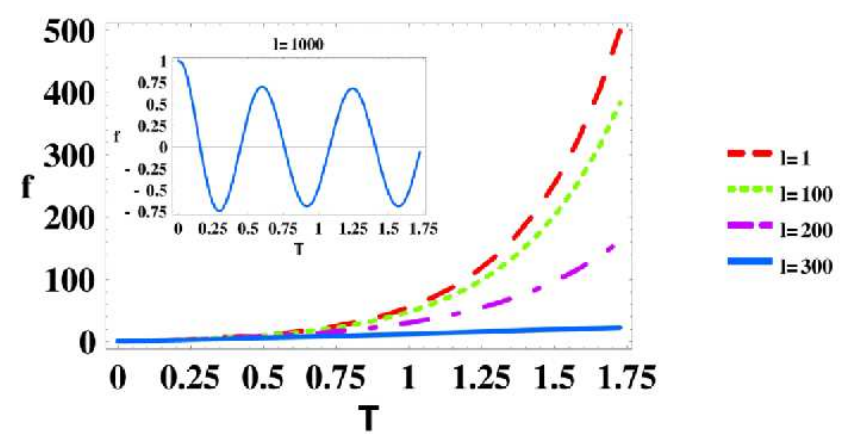

FIG. 14: $f\left(l, z_{0}=.5, Q=-10^{-4}, T\right)$ for various $l$. The inset shows the oscillatory behavior of $f$ for large $l$.

and therefore set the typical initial amplitudes of the mode functions as the r.m.s. value $\left\langle\Phi_{\mathrm{lm}}^{2}\right\rangle^{1 / 2}$ from Eq. 53 The velocity field can be decomposed into spherical harmonics just as $\Phi$ was, and the analysis performed above carries over exactly. The typical initial size of the velocity mode functions is then given by

$$
\left\langle\Phi_{\operatorname{lm}}^{\prime 2}\right\rangle=\frac{4 \pi A_{l}}{2 l+1} .
$$

with

$$
A_{l}=\frac{2 l+1}{4 \pi} \int_{-1}^{1} d(\cos \Psi)\left\langle\Phi^{\prime} \tilde{\Phi}^{\prime}\right\rangle P_{l}(\cos \Psi) .
$$

The initial amplitudes in Eq. 53 and Eq. $[55]$ can now be evolved to the turning point, and the mode functions resummed. The ensemble average of the r.m.s. fluctuations in $\Phi$ at any time at a given point will then be:

$$
\begin{aligned}
\left\langle\Phi(T)^{2}\right\rangle & =\sum_{l} \frac{2 l+1}{4 \pi}\left\langle\Phi_{\operatorname{lm}}(T)^{2}\right\rangle \\
& =\sum_{l}\left[C_{l}^{1 / 2} f\left(l, z_{0}, q, T\right)+A_{l}^{1 / 2} g\left(l, z_{0}, q, T\right)\right]^{2},
\end{aligned}
$$


which can be evaluated at $T=T_{\max }$.

A full model of the two-point functions $\langle\Phi \tilde{\Phi}\rangle$ and $\left\langle\Phi^{\prime} \tilde{\Phi}^{\prime}\right\rangle$ would involve quantizing the mode functions on the curved spacetime of the bubble wall worldsheet, which has a metric depending on $z(T)$. Further, to treat large fluctuations, we would need to include non-linear terms in the equation of motion. The exact two-point function is therefore a rather formidable object to compute. As a simplified model, we will employ the two-point functions of a massless scalar field in flat spacetime, and replace the spatial distance $r$ with the distance along the bubble wall $r_{0} \Psi$. This massless scalar corresponds to the perturbations on a flat wall separating domains of equal energy density in Minkowski space [31]. Corrections to this picture in the presence of curvature should be small over small regions of the bubble wall. We are also neglecting the large difference in energy densities across the bubble wall, which will give the field a (negative) mass to first order. The apparent divergence of the correlator due to this negative mass will be rendered finite by the nonlinear terms which must be introduced to discuss large fluctuations. In light of all these difficulties, and several more approximations we will make below, this should be considered as a first, rough estimate of the amplitude of the quantum fluctuations on the bubble at the time of nucleation.

The space-like two-point function in Minkowski space at large separations is given by

$$
\langle\phi(\mathbf{x}) \phi(\mathbf{y})\rangle=\frac{\sigma^{-1}}{4 \pi r}
$$

where $r \equiv|\mathbf{x}-\mathbf{y}|$. As in the work of Garriga and Vilenkin [31], we introduce a smeared field operator to obtain a well-defined answer at close separations

$$
\phi_{s} \equiv \frac{1}{\pi s^{2}} \int_{|\mathbf{y}-\mathbf{x}|<\mathbf{s}} d^{2} y \phi(\mathbf{y})
$$

where $s$ is a smearing length, chosen to be the Compton wavelength $s=\sigma^{-1 / 3}$ of a piece of wall, which is a physically reasonable lower bound on the size of a measurable region. The smeared correlator will then be given by

$$
\begin{aligned}
\left\langle\phi_{s}(\mathbf{x}) \phi_{s}(\mathbf{y})\right\rangle=\frac{1}{\pi^{2} s^{4}} & \int_{|\mathbf{z}-\mathbf{x}|<\mathrm{s}} d^{2} z \\
& \int_{|\mathbf{q}-\mathbf{y}|<\mathrm{s}} d^{2} q\langle\phi(\mathbf{z}) \phi(\mathbf{q})\rangle,
\end{aligned}
$$

which evaluates at $\mathbf{x}=\mathbf{y}$ to

$$
\left\langle\phi_{s}^{2}\right\rangle=\left(\frac{1}{2}+\mathcal{G}\right) \sigma^{-2 / 3},
$$

where $\mathcal{G}=.916 \ldots$ is Catalin's constant. We have been unable to integrate Eq. 60 to obtain the exact form of the smeared two-point function for all $r$. However, it must smoothly interpolate between the value at $r=0$ in Eq. 61] to the functional form at $r \gg s$ given by Eq. 58
We therefore employ the following 'toy model' smeared field correlator

$$
\begin{aligned}
\langle\phi(\mathbf{x}) \phi(\mathbf{y})\rangle= & \frac{\sigma^{-1}}{4 \pi s}\left[(2 \pi+4 \pi \mathcal{G}-1) e^{-r^{2} / 2 s^{2}}\right. \\
& \left.+\frac{1}{r / s+1}\right]
\end{aligned}
$$

which has the correct asymptotics. With $r=r_{0} \Psi$, the dimensionless form of the toy correlator is given by:

$$
\begin{gathered}
\langle\Phi \tilde{\Phi}\rangle=\frac{(-Q)}{(24 \pi)^{2}} \quad\left[(2 \pi+4 \pi \mathcal{G}-1) e^{-\left(R^{2} / 2\right) \Psi^{2}}\right. \\
\left.+\frac{1}{R \Psi+1}\right]
\end{gathered}
$$

where $R \equiv r_{0} / s$.

The velocity-velocity correlator can be calculated using the Hamiltonian approach. For spacelike separations, this is given by

$$
\begin{aligned}
\left\langle\phi^{\prime}(\mathbf{x}) \phi^{\prime}(\mathbf{y})\right\rangle & =\sigma^{-1} \int \frac{|p| d^{2} p}{2(2 \pi)^{2}} \exp [i \mathbf{p}(\mathbf{x}-\mathbf{y})] \\
& =\sigma^{-1} \int_{0}^{\Lambda} \frac{d p}{4 \pi} p^{2} J_{0}(p r)
\end{aligned}
$$

where we have introduced a hard momentum cut-off $\Lambda$ to obtain a finite answer. This cutoff will correspond to the inverse smearing length, with higher momentum scales higher accounted for in the smeared operator. The integral can be evaluated in terms of generalized hypergeometric functions as

$$
\left\langle\phi^{\prime}(\mathbf{x}) \phi^{\prime}(\mathbf{y})\right\rangle=\frac{\Lambda^{3} \sigma^{-1}}{12 \pi}{ }_{1} F_{2}\left(\frac{3}{2} ; 1, \frac{5}{2} ;-\frac{\Lambda^{2} r^{2}}{4}\right) .
$$

At close separations, we construct a smeared operator $\phi_{s}^{\prime}$. The expectation value of this operator at zero separation is

$$
\left\langle\phi_{s}^{\prime 2}\right\rangle=\frac{\log (64)+2 \gamma-4}{2 \pi^{2}},
$$

where $\gamma=.577 \ldots$ is the Euler-Mascheroni constant. Smoothly connecting the small $r$ (Eq. 66) and large $r$ (Eq. 65) behavior as in Eq. 62 the $\Phi^{\prime}$ (defined similarly to $z^{\prime}$ ) correlator on the bubble at the time of nucleation is:

$$
\begin{aligned}
\left\langle\Phi^{\prime} \tilde{\Phi}^{\prime}\right\rangle= & \frac{(-Q)}{12 \pi}\left[{ }_{1} F_{2}\left(\frac{3}{2} ; 1, \frac{5}{2} ;-\frac{R^{2} \Psi^{2}}{4}\right)\right. \\
& \left.+\left(\frac{12 \gamma-6 \log (64)-24}{\pi}-1\right) e^{-\left(R^{2} / 2\right) \Psi^{2}}\right]
\end{aligned}
$$

The integrals Eq. 54 and Eq. 56 for the correlators Eq. 63] and Eq. 67 must be evaluated numerically. Calculation of the coefficients for every $l$ and $R$ is unfortunately unfeasible because of the highly oscillatory behavior of the integrands and sheer number of mode functions 
that must be considered. However, we have been able to deduce sufficiently good approximate fits for $C_{l}$ and $A_{l}$ as a function of both $l$ and $R$. In both cases, the power is dominated by a peak at $l \simeq R$.

The $C_{l}$ are nicely fit by the function

$$
\begin{aligned}
C_{l}= & \frac{(-Q)}{(24 \pi)^{2}} \frac{83}{100} \frac{\sqrt{2} l+1}{\sqrt{2} R+1} \\
& \exp \left\{\frac{-1}{4 R^{2}-2}\left[(\sqrt{2} l+1)^{2}-(\sqrt{2} R+1)^{2}\right]\right\} .
\end{aligned}
$$

The proposed fit for the $A_{l}$ consists of two power laws matched at the $R=l$ peak. For $l \leq R$, the best fit is $A_{l}=(-Q) l^{5 / 4} /\left(10 R^{9 / 4}\right)$ and for $\bar{l}>R$, the fit is $A_{l}=3(-Q) R^{1.8} /\left(200 l^{2.6}\right)$. Because these power law indices are slightly uncertain, we only count the modes with $l \leq R$ in the $A_{l}$. This is conservative, and also justified because these modes will not contribute significantly to the sum in Eq. 57.

With these initial conditions, we can now evolve each mode function using Eq. 49 and then re-sum in Eq. 57 to find the average size of the fluctuations at the turning point. We have calculated $f$ and $g$ up to the $l$ corresponding to the last unstable mode of the smallest $|Q|$, for all three inflation scales $\left(M_{I}=100 \mathrm{GeV}, M_{I}=10^{14} \mathrm{GeV}\right.$, and $M_{I}=10^{17} \mathrm{GeV}$ ). The results for weak- and GUTscale inflation are shown in Fig. 13] where the dotted line indicates the boundary of the region over which the perturbations become non-linear (non-linear to the left of the line). It can be seen that in this model, even just quantum perturbations of the bubble wall grow nonlinear in bubbles that start at radii less than about one-tenth of the turnaround radius; this grossly violates the assumption of spherical symmetry used in tunneling calculations. On the other hand, none of the parameter space in the high inflation scale case went non-linear, and at all scales there is always a region of initial bubble radii near the turnaround radius, for which nonlinearity never occurs.

\section{Thick Walls and Radiation}

Just as we have no reason to expect a fluctuated region to be spherically symmetric, we have no reason to assume that it will have thin walls. An analysis of thick-walled true vacuum bubbles was undertaken in Ref. [31, 32], where it was found that the instabilities found in thinwalled case are still present in the form of deformations normal to the bubble profile. In the case of small false vacuum bubbles, there is no obvious consideration (such as a corresponding instanton) to supply the profile of the bubble wall, and so we can merely conjecture by precedent that the instability would be retained in the thickwalled case as well.

Another consideration, applying to bubbles smaller than the false vacuum horizon size, is whether inflation is spoiled by non-vacuum contributions to the energy density. The perturbations on the bubble wall translate into gravitational waves [33, 34], and since the bound bubble solutions remain relatively close to their gravitational radius and become distorted over many different length scales on a relatively short time scale (see the quasiexponential growth in Fig. 14), they will be emitters of copious gravitational radiation. Another problem arises if the kinetic and gradient energy of the field becomes appreciable in the bubble interior, either from intrusion of the wall (for example, imagine a bubble being pinched in half by some non-linear perturbation), or from particle production or other scalar modes propagating in from the wall. If the emission of energy into the interior of the bubble from any combination of these modes makes a significant contribution to the equation of state, then inflation will not occur.

\section{SUMMARY \& DISCUSSION}

In this paper we have examined the feasibility of producing inflationary universes from a spacetime with a small cosmological constant. Reviewing the solutions allowed by the junction conditions, there are several distinct possibilities if one allows for violations of the null energy condition. The first is the Farhi, Guth \& Guven (FGG) mechanism, in which (referring to Fig. 9 and Fig. (10) the IV-I-II/III (solution 1) or IV-III-II/III (solution 2) solution is fluctuated in the expanding phase and then tunnels to an unbound solution, as shown in Fig.11] To the outside observer in region I of the SdS conformal diagram it appears that the bubble has disappeared behind the horizon, but on the other side of the wormhole, both an inflationary universe and a non-inflating universe (an asymptotically true-vacuum de Sitter region) have come into existence. In one case, shown in solution 5 of Fig. 10 we can imagine an observer in region III, then take the Schwarzschild mass $m \rightarrow 0$ limit (thus removing regions I, II and IV entirely) and interpret the spacetime as that of the analytically continued Coleman-De Luccia instanton. Since the very same spacetime takes part in both the FGG mechanism and the Coleman-De Luccia false vacuum instanton, there may be some way to smoothly interpolate between these two processes; we intend to explore this idea further in future work.

In addition to the FGG and regular instanton mechanisms, there are two more possibilities. First, an inflationary universe might be directly produced by some null energy condition (NEC) violating fluctuation into one of the unbound or monotonic solutions shown in Solutions 5-13 of Fig. 10. To the outside observer in region I of the SdS conformal diagram, these solutions would be indistinguishable from the fluctuation of a black hole, but they would secretly entail the creation of everything on the other side of the wormhole, as in the FGG tunneling. A second method of direct production is the fluctuation (which does not require an NEC violation) of the IVI-II'/III-II solution (solution 3 and 4). These solutions only exist in the case where the interior and exterior cos- 
mological constants are comparable, and so would not correspond to the nucleation of inflation from a universe like ours, but they might be of interest in understanding transitions between nearly degenerate vacua.

Examining these classical solutions to first order, we have shown that an instability to aspherical perturbations exists in those solutions which possess a turning point. This includes the bound (solutions 1 and 2) and unbound solutions (solutions 5-9). In the latter case there is no clear way to set an initial radius or initial perturbation amplitude, so we can say only that collapsing bubbles are violently unstable. The bound solutions are amenable to quantitative investigation, and we have focused on the growth of perturbations in the expanding phase that precedes tunneling in the FGG mechanism. For bound expanding bubbles formed by the fluctuations of a scalar field in de Sitter space, there is no instanton to enforce spherical symmetry, so we would expect initial aspherical perturbations to be relatively large. Since there is no detailed model for the fluctuating scalar field to see how large, we have instead calculated an estimate of the minimal deviations from spherical symmetry in light of quantum fluctuations, and present this as an extremely rare, best case scenario for spherical symmetry. These minimal fluctuations were then evolved to the turning point of the bound solutions, which is the point in the FGG mechanism where there is a chance for tunneling to an inflationary universe to occur. Of the three representative energy scales for inflation (false vacuum energy densities) we have studied, the evolved minimal perturbations on a Plank scale bubble remain small over most of the allowed parameter space, while the perturbations on GUT and weak scale bubbles can grow nonlinear if they start at a sufficiently small $(\lesssim 10 \%)$ fraction of the turnaround radius. Thus even in the best-case scenario some bubbles become nonlinear, but on the other hand there will always in principle be some that do not.

The instability introduces complications into the use of the FGG mechanism as a means of baby universe production. The existing calculations of the tunneling rate rely heavily on the assumption of spherical symmetry. It is unclear how to perform a similar calculation for a (possible non-linearly) perturbed bubble, as the number of degrees of freedom has drastically increased and the assumption of a minisuperspace of spherically symmetric metrics is no longer good. Further, the bubble interior will become filled with scalar gradient and kinetic energy and gravity waves, possibly upsetting the interior sufficiently to prevent inflation. One might argue that in an eternal universe there is plenty of time to wait around for a fluctuation which is sufficiently spherical. However, to fully understand the importance of the FGG mechanism, one must both have a model of the scalar field fluctuations, which would predict the distribution of bubble shapes and masses, and also a model for tunneling in the presence of asphericities. In addition, one must understand the competition among the various processes which result in the formation of inflating false vacuum regions, and thus show that the FGG mechanism is not a relative rarity. These problems represent significant calculations in a theory of quantum gravity which we do not yet possess, but progress in these areas would undoubtedly improve our understanding of the initial conditions for inflation.

\section{Acknowledgments}

The authors wish to thank A. Albrecht, T. Banks, P.J. Fox, S. Gratton, and T. Mai for their assistance in the development of this work.
[1] E. Farhi and A. H. Guth, Phys. Lett. B 183, 149 (1987).

[2] E. Farhi, A. H. Guth and J. Guven, Nucl. Phys. B 339, 417 (1990).

[3] W. Fischler, D. Morgan and J. Polchinski, Phys. Rev. D 41, 2638 (1990); W. Fischler, D. Morgan and J. Polchinski, Phys. Rev. D 42, 4042 (1990).

[4] S. K. Blau, E. I. Guendelman and A. H. Guth, Phys. Rev. D 35, 1747 (1987).

[5] L. Dyson, M. Kleban and L. Susskind, JHEP 0210, 011 (2002) arXiv:hep-th/0208013.

[6] A. Albrecht and L. Sorbo, Phys. Rev. D 70, 063528 (2004) arXiv:hep-th/0405270.

[7] S. M. Carroll and J. Chen, arXiv:hep-th/0410270

[8] S. Hollands and R. M. Wald, Gen. Rel. Grav. 34, 2043 (2002) arXiv:gr-qc/0205058.

[9] R. Penrose, in "Fourteenth Texas Symposium on Relativistic Astrophysics, Dallas, Tex., 1988" (New York: Annales of the New York Academy of Sciences, 1989)

[10] K. M. Lee and E. J. Weinberg, Phys. Rev. D 36, 1088 (1987).
[11] R. Basu, A. H. Guth and A. Vilenkin, Phys. Rev. D 44, 340 (1991); J. Garriga, Phys. Rev. D 49, 6327 (1994) arXiv:hep-ph/9308280.

[12] T. Banks, arXiv:hep-th/0211160

[13] R. Bousso, Phys. Rev. D 71, 064024 (2005) arXiv:hep-th/0412197.

[14] S. Dutta and T. Vachaspati, Phys. Rev. D 71, 083507 (2005) arXiv:astro-ph/0501396.

[15] T. Vachaspati and M. Trodden, Phys. Rev. D 61, 023502 (2000) arXiv:gr-qc/9811037.

[16] V. A. Berezin, V. A. Kuzmin and I. I. Tkachev, Phys. Lett. B 120, 91 (1983); V. A. Berezin, V. A. Kuzmin and I. I. Tkachev, Phys. Rev. D 36, 2919 (1987).

[17] H. Sato, Prog. Theor. Phys. 76, 1250 (1986).

[18] A. Aurilia, M. Palmer and E. Spallucci, Phys. Rev. D 40, 2511 (1989).

[19] K. Lake and R. C. Roeder, Phys. Rev. D 15, 3513 (1977).

[20] T. R. Choudhury and T. Padmanabhan, arXiv:gr-qc/0404091

[21] S. R. Coleman, Phys. Rev. D 15, 2929 (1977) [Erratum- 
ibid. D 16, 1248 (1977)].

[22] C. G. . Callan and S. R. Coleman, Phys. Rev. D 16, 1762 (1977).

[23] S. R. Coleman and F. De Luccia, Phys. Rev. D 21, 3305 (1980).

[24] R. Penrose, Phys. Rev. Lett. 14, 57 (1965).

[25] A. D. Linde, Nucl. Phys. B 372, 421 (1992) arXiv:hep-th/9110037.

[26] J. Garriga and A. Megevand, Int. J. Theor. Phys. 43, 883 (2004) arXiv:hep-th/0404097.

[27] A. Gomberoff, M. Henneaux, C. Teitelboim and F. Wilczek, Phys. Rev. D 69, $083520 \quad$ (2004) arXiv:hep-th/0311011.

[28] F. C. Adams, K. Freese and L. M. Widrow, Phys. Rev. D 41, 347 (1990).

[29] J. Garriga and A. Vilenkin, Phys. Rev. D 44, 1007 (1991).

[30] J. Guven, Phys. Rev. D 48, 4604 (1993) arXiv:gr-qc/9304032.

[31] J. Garriga and A. Vilenkin, Phys. Rev. D 45, 3469 (1992).

[32] T. Vachaspati and A. Vilenkin, Phys. Rev. D 43, 3846 (1991).

[33] A. Ishibashi and H. Ishihara, Phys. Rev. D 56, 3446 (1997) arXiv:gr-qc/9704058.

[34] A. Ishibashi and H. Ishihara, Phys. Rev. D 60, 124016 (1999) arXiv:gr-qc/9802036.
[35] We will include the means by which the constructable expanding bubble is produced, be it in the lab or by some quantum or thermal fluctuation, in the definition of the Farhi-Guth-Guven mechanism

[36] This, in fact, connects to a more general question of whether inflation can really be said to have "general" initial conditions, given that it must be low entropy; see, e.g., 7, 8, 9].

[37] We follow the methods and notations of these works, though they are not the only on the subject.

[38] Ref. [18] considered only the case where the sign change occurred to the left of the maximum.

[39] Many, but not all, of these solutions have appeared previously in the literature [1, 16, 18]

[40] There are anti-trapped surfaces in region II' of the SdS diagram, and there is a noncompact Cauchy surface $C$ for it, so the Penrose theorem applies, but only indicates that geodesics are incomplete in region II' because they reach its edge (the past Cauchy horizon of $C$.) The region is thus extendible (into regions I, III' and IV') rather than singular. Full dS has only compact Cauchy surfaces so the theorem does not apply.

[41] The mass of a piece of wall of scale $s$ is $M \simeq s^{2} \sigma$, where $\sigma$ is the wall surface energy density; the Compton wavelength is then found by setting $M=s^{-1}$, yielding $s=z_{\text {compton }} \simeq \sigma^{-1 / 3}$. 